\title{
Tabela de vida de fertilidade de Lysiphlebus testaceipes (Cresson) (Hymenoptera, Braconidae, Aphidiinae) em Rhopalosiphum maidis (Fitch) e Aphis gossypii Glover (Hemiptera, Aphididae)
}

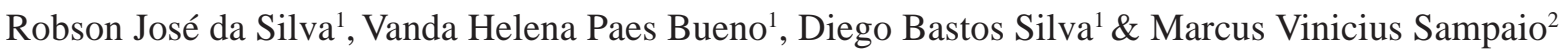

${ }^{1}$ Laboratório de Controle Biológico, Universidade Federal de Lavras, Departamento de Entomologia, Caixa Postal 3037, 37200-000, Lavras-MG, Brasil.ecosbio@yahoo.com.br, vhpbueno@ufla.br

${ }^{2}$ Instituto de Ciências Agrárias, Universidade Federal de Uberlândia, Campus Umuarama. Caixa Postal 593, 38400-902. Uberlândia-MG, Brasil. mvsampaio@iciag.ufu.br

\begin{abstract}
Fertility life table of Lysiphlebus testaceipes (Cresson) (Hymenoptera, Braconidae, Aphidiinae) in Rhopalosiphum maidis (Fitch) and Aphis gossypii Glover (Hemiptera, Aphididae). The evaluation of the growth potential of Lysiphlebus testaceipes (Cresson) is important for its use in biological control programs of aphids. This work aimed to evaluate the fertility life table of L. testaceipes in Rhopalosiphum maidis (Fitch) and Aphis gossypii Glover. To determine the immature mortality, development and the sex ratio of the parasitoid, 12 females parasitoid, and 480 nymphs of each aphids were used. To evaluate the longevity and fertility 15 female parasitoid were used. Nymphs of each aphid ( 3 day old) were offered for each parasitoid female daily, until the female died, being 300 ( $1^{\text {st }}$ day); 250 ( $2^{\text {nd }}$ day); 200 ( $3^{\text {rd }}$ day); 150 ( $4^{\text {th }}$ day) and 50 nymphs in the other days. L. testaceipes showed immature mortality rates of $5.6 \%$ in $R$. maidis and $9.2 \%$ in A. gossypii. The development time of L. testaceipes in R. maidis and A. gossypii was 10.2 and 10.1 days, and the sex ratio of 0.71 and 0.66 , respectively. The female of L. testaceipes had a fecundity of 498.8 eggs in $R$. maidis and 327.8 eggs in A. gossypii. The growth parameters the L. testaceipes in $R$. maidis and A. gossypii were, respectively $\mathrm{R}_{\mathrm{O}}=205.38$ and 164.08 females; $\mathrm{r}_{\mathrm{m}}=0.449$ and 0.431 females/females/day; $\ddot{e}=1.57$ and 1.54 females/day; $\mathrm{T}=11.86$ and 11.83 days and $\mathrm{TD}=10.78$ and 11.27 days. L. testaceipes showed great growth potential on both aphid hosts. $R$. maidis could be a suitable host for proposals of mass-rearing and open rearing system using $L$. testaceipes.
\end{abstract}

KEYWORDS. Growth parameters; longevity; parasitoid; reproductive rate.

RESUMO. Tabela de vida de fertilidade de Lysiphlebus testaceipes (Cresson) (Hymenoptera, Braconidae, Aphidiinae) em Rhopalosiphum maidis (Fitch) e Aphis gossypii Glover (Hemiptera, Aphididae). Avaliar o potencial de crescimento de Lysiphlebus testaceipes (Cresson) é importante para seu uso em programas de controle biológico de pulgões. Este trabalho teve como objetivo determinar a tabela de vida de fertilidade de L. testaceipes em Rhopalosiphum maidis (Fitch) e Aphis gossypii Glover. Na avaliação da mortalidade de imaturos, do desenvolvimento e da razão sexual foram utilizadas 12 fêmeas do parasitóide e 480 ninfas de cada pulgão testado. Na avaliação da longevidade e da fertilidade foram utilizadas 15 fêmeas do parasitóide e uma colônia por dia de cada pulgão, até a morte da fêmea do parasitóide, sendo 300 ( $\left.1^{\circ} \mathrm{dia}\right) ; 250\left(2^{\circ} \mathrm{dia}\right) ; 200\left(3^{\circ} \mathrm{dia}\right) ; 150\left(4^{\circ} \mathrm{dia}\right) ; 100$ ( $\left.5^{\circ} \mathrm{dia}\right)$ e 50 ninfas nos demais dias. L. testaceipes apresentou taxas de mortalidade de imaturos de 5,6\% em $R$. maidis e de 9,2\% em A. gossypii, desenvolvimento de 10,2 e 10,1 dias e razão sexual de 0,71 e 0,66 , respectivamente. L. testaceipes apresentou fecundidade de 498,2 ovos em $R$. maidis e de 327,8 ovos em A. gossypii. Os parâmetros de crescimento de L. testaceipes em $R$. maidis e A. gossypii foram, respectivamente, $\mathrm{R}_{\mathrm{O}}=205,38$ e 164,08 fêmeas; $\mathrm{r}_{\mathrm{m}}=0,449$ e 0,431 fêmeas/fêmeas/dia; $\lambda=1,57$ e 1,54 fêmeas/dia; $\mathrm{T}=11,86$ e 11,83 dias e $\mathrm{TD}=10,78$ e 11,27 dias. L. testaceipes apresenta alto potencial de crescimento em $R$. maidis e A. gossypii. $R$. maidis mostrou ser hospedeiro adequado aos propósitos de criação massal e utilização em sistema de criação aberta para $L$. testaceipes.

PALAVRAS-CHAVE. Longevidade; parâmetros de crescimento; parasitóide; taxa reprodutiva.

Pulgões são pragas importantes em diversas culturas, tanto em condições de campo como em cultivos protegidos. São estrategistas $r$ muito bem adaptados às explorações de um habitat novo e temporário devido à sua alta capacidade reprodutiva e são, ainda, responsáveis por danos diretos devido a grande sucção de seiva e indiretos, como as transmissões de vírus as plantas (Bueno 2005).

O pulgão Rhopalosiphum maidis (Fitch, 1856) é especialista em Poaceae, estando relacionado a mais de 30 gêneros nesta família, especialmente em cultivos de cereais (Kuo et al. 2006). No Brasil, é encontrado principalmente em regiões onde se cultivam o sorgo e o milho "safrinha", causando danos econômicos (Fonseca et al. 2004). Espécies como R. maidis e R. padi (Linnaeus, 1758) são importantes no uso em sistemas de criação aberta, ou seja, na conservação de parasitóides como Lysiphlebus testaceipes (Cresson, 1880). Populações de Aphidius spp. foram mantidas continuamente por meio desse sistema para o controle de pulgões-praga em cultivos de crisântemo (Ramakers \& O’Neill 1999; Ramakers \& Maaswinkel 2002).

Aphis gossypii Glover, 1877 é uma espécie cosmopolita de grande importância econômica e a mais comum em cultivos de 
curcubitáceas como o pepino e plantas ornamentais como o crisântemo (Bueno 2005; Carvalho et al. 2006; Soglia et al. 2006). Fêmeas de $A$ gossypii podem produzir de 3 a 10 ninfas por dia e, assim, o seu crescimento populacional pode ser extremamente rápido. Em pepino, a população desse pulgão pode aumentar 12 vezes em sete dias (Malais \& Ravensberg 2003).

Tanto R. maidis como A. gossypii são hospedeiros de parasitóides afidiíneos, entre eles o endoparasitóide solitário L. testaceipes, uma das espécies mais comuns na América do Sul (Starý et al. 2007) e com grande potencial para ser usado em liberações inoculativas sazonais (Rodrigues et al. 2005) e na manutenção em sistemas de criação aberta (Rodrigues et al. 2001) em programas de controle biológico de afídeos. É responsável por taxas de parasitismo acima de $50 \%$ em $A$. gossypii (Rodrigues \& Bueno 2001; Rodrigues et al. 2004), apresenta grande eficiência no controle natural de $R$. maidis em cultivo de trigo (Alves et al. 2005) e é capaz de colonizar casas de vegetação espontaneamente em áreas do Mediterrâneo na Europa (Rochat 1997) e também no Brasil (Bueno et al. 2003b).

Parasitóides oligófagos, isto é, espécies que podem atacar pulgões pertencentes a gêneros relacionados, são geralmente escolhidas para programas de controle biológico aplicado. No entanto, o sucesso de seu uso depende, entre outras coisas, da sua produção eficiente e econômica no laboratório. Por outro lado, o sucesso dessa criação depende da utilização de dados do seu ciclo de vida em espécies hospedeiras adequadas; neste sentido, as tabelas de vida são importantes instrumentos para a compreensão e determinação do crescimento populacional das espécies (Bellows-Junior et al. 1992; Van Lenteren \& Woets 1988). Segundo Van Lenteren (2000), a taxa intrínseca de aumento $\left(\mathrm{r}_{\mathrm{m}}\right)$, importante parâmetro obtido por meio da tabela de vida, é um fator a ser considerado na observação da efetividade de um parasitóide como agente de controle biológico.

Assim, embora trabalhos venham demonstrando o potencial de L. testaceipes, ainda são necessários estudos que avaliem sua capacidade reprodutiva em hospedeiros adequados, o que subsidia o conhecimento quanto às possibilidades de sua criação massal e seu uso em sistemas de criação aberta para, conseqüentemente, contribuir na comprovação de sua efetividade como agente de controle em programas de controle biológico de afídeos.

\section{MATERIALE MÉTODOS}

O potencial de crescimento do parasitóide L. testaceipes foi avaliado nos hospedeiros $R$. maidis e A. gossypii em câmara climática à temperatura de $25 \pm 1^{\circ} \mathrm{C}, 70 \pm 10 \%$ de UR e $12 \mathrm{~h}$ de fotofase. Foram analisados os parâmetros mortalidade de imaturos, desenvolvimento, reprodução e longevidade das fêmeas.

Criação dos pulgões. Os pulgões R. maidis e A. gossypii foram obtidos em plantas de sorgo [Sorghum bicolor (L.) Moench var. BR 303] e pepino (Cucumis sativus L. var. caipira), respectivamente, no Campus da Universidade Federal de Lavras (UFLA), e multiplicados no Laboratório de Controle Biológico do Departamento de Entomologia da mesma instituição em plantas de sorgo e pepino, respectivamente. Fêmeas adultas ápteras de cada espécie foram transferidas para placas de Petri (15 cm de diâmetro) contendo discos foliares de sorgo e pepino, de acordo com a espécie do pulgão, sobre uma camada de ágar-água $1 \%$, vedadas com papel toalha. As placas foram mantidas em câmara climática e, após 24 horas, as fêmeas adultas foram retiradas, restando apenas as ninfas que, com três dias de idade, foram oferecidas às fêmeas do parasitóide L. testaceipes.

Obtenção dos parasitóides. Os parasitóides L. testaceipes foram provenientes da criação de manutenção do Laboratório de Controle Biológico do Departamento de Entomologia da Universidade Federal de Lavras (UFLA), os quais foram mantidos no pulgão Schizaphis graminum (Rondani, 1852) em folhas de sorgo durante várias gerações. Fêmeas e machos obtidos dessa criação foram acasalados e liberados em placas de Petri (15 cm de diâmetro) contendo secções de folhas de sorgo fixadas em uma camada de ágar-água a $1 \%$ e ninfas de $2^{\circ}$ e $3^{\circ}$ ínstares de S. graminum. Múmias formadas nessas placas foram individualizadas em tubos de vidros (100 $\mathrm{mm}$ x $8 \mathrm{~mm}$ ) contendo gotículas de água e mel, tampados com filme PVC e mantidos em câmara climática até a emergência dos parasitóides. Após a emergência, machos e fêmeas foram separados e sexados, e posteriormente acasalados para a utilização nos experimentos.

Mortalidade de imaturos e desenvolvimento de $L$. testaceipes. Para obtenção das taxas de mortalidade de imaturos e desenvolvimento foram utilizadas 12 fêmeas acasaladas de L. testaceipes, com menos de $24 \mathrm{~h}$ de vida, sem experiência prévia de oviposição e alimentadas com gotículas de mel e água, e 480 ninfas de cada um dos pulgões, $R$. maidis e $A$. gossypii.

Para cada espécie de pulgão testado foram utilizadas placas de Petri ( $6 \mathrm{~cm}$ de diâmetro) contendo 40 ninfas com três dias de idade sobre disco foliar de sorgo com $R$. maidis e disco foliar de pepino com A. gossypii em uma camada de ágar-água $1 \%$. Uma fêmea do parasitóide $L$. testaceipes foi liberada em cada placa, permanecendo por 20 minutos contados a partir da primeira oviposição realizada pela fêmea. Ao final desse período as fêmeas foram retiradas e as placas, mantidas em câmara climática. Após três dias do parasitismo, 50\% do número total de pulgões de cada placa foram retirados e dissecados para a contagem do número de larvas do parasitóide; os outros $50 \%$ foram mantidos até a formação das múmias. As múmias formadas foram individualizadas em tubos de vidro $(100 \mathrm{~mm} \mathrm{x}$ $8 \mathrm{~mm}$ ) contendo gotículas de mel e água, vedados com filme PVC. Foram avaliados o período de desenvolvimento (da oviposição á emergência) e a razão sexual dos parasitóides em cada pulgão hospedeiro testado.

A mortalidade de imaturos foi estimada pela diferença entre o número de adultos emergidos e o número de larvas encontradas em cada espécie de pulgão.

Reprodução e longevidade de $L$. testaceipes. Para avaliação 
dos parâmetros de reprodução e longevidade foram utilizadas 15 fêmeas acasaladas do parasitóide L. testaceipes, com menos de 24 horas de idade, alimentadas com mel e água.

Para cada fêmea do parasitóide foi oferecida uma colônia com ninfas de $R$. maidis ou A. gossypii com três dias de idade, em placa de Petri ( $15 \mathrm{~cm}$ de diâmetro) contendo discos foliares de pepino ou sorgo, de acordo com a espécie do pulgão, sobre uma camada de ágar-água $1 \%$, vedadas com papel toalha. Até a morte da fêmea do parasitóide foi oferecida uma nova colônia de $R$. maidis ou A. gossypii por dia. Foram utilizadas, para cada fêmea do parasitóide durante seu período de vida, as seguintes densidades de cada espécie do pulgão: $1^{\circ}$ dia -300 ninfas; $2^{\circ}$ dia -250 ninfas; $3^{\circ}$ dia -200 ninfas; $4^{\circ}$ dia -100 ninfas; e nos dias seguintes, 50 ninfas até a morte da fêmea.

As placas contendo as ninfas parasitadas foram mantidas em câmara climática por três dias, para o desenvolvimento das larvas do parasitóide. Após esse período, as ninfas foram transferidas para um "freezer" a $5^{\circ} \mathrm{C}$, para promover a paralisação do desenvolvimento da larva. A quantidade de ovos colocados por fêmea do parasitóide foi estimada em função do número de larvas dentro de indivíduos de cada espécie de pulgão hospedeiro, os quais foram dissecados com o auxílio de estilete, sob microscópio estereoscópico, em solução de cloreto de sódio 1\%, conforme Steenis (1994) e Rodrigues et al. (2003).

Análise dos dados. Para construção da tabela de vida de fertilidade de L. testaceipes, foi utilizado o ponto médio de cada idade das fêmeas parentais do parasitóide $(\mathbf{x})$, contada a partir da fase de ovo; a expectativa de vida até a idade $\mathrm{x}\left(\mathbf{l}_{\mathbf{X}}\right)$; a fertilidade específica $\left(\mathbf{m}_{\mathbf{x}}\right)$ e o número total de fêmeas nascidas na idade $\mathrm{x}\left(\mathbf{l}_{\mathbf{x}} \mathbf{m}_{\mathbf{x}}\right)$.

Os parâmetros de crescimento da população do parasitóide foram calculados com base na tabela de vida de fertilidade, sendo $\mathbf{R}_{\mathbf{O}}=$ taxa líquida de reprodução; $\mathbf{T}=$ tempo médio de geração; $\mathbf{r}_{\mathbf{m}}=$ taxa intrínseca de aumento; $\lambda=$ razão finita de aumento; e $\mathbf{T D}=$ tempo que leva a população para duplicar em número.

\section{RESULTADOSEDISCUSSÃO}

Mortalidade de imaturos e desenvolvimento de $\boldsymbol{L}$. testaceipes em $\boldsymbol{R}$. maidis e A. gossypii. A mortalidade de imaturos de L. testaceipes em $R$. maidis e A. gossypii foi de 5,6 e $9,2 \%$, respectivamente, valores inferiores aos encontrados por Rodrigues et al. (2003) (22,2\%) quando L. testaceipes teve como hospedeiro $S$. graminum. Steenis (1994) obteve $29,6 \%$ de mortalidade de L. testaceipes em A.gossypii, valor superior ao encontrado neste trabalho. No presente trabalho, a metodologia proposta por Steenis (1994) e Rodrigues et al. (2003) foi modificada, sendo oferecido para as fêmeas de $L$. testaceipes um maior número de pulgões e utilizado um maior tempo de exposição destes hospedeiros à fêmea do parasitóide. É provável que essas alterações na metodologia tenham contribuído para melhorar a acuidade na obtenção dos dados de mortalidade do parasitóide.

Esta baixa mortalidade observada também pode ser devida ao fato de que os pulgões $R$. maidis e $A$. gossypii apresentam maior qualidade ao desenvolvimento das formas imaturas do parasitóide L. testaceipes. Segundo Sequeira \& Mackauer (1993) e Mackauer et al. (1996), para que uma fêmea de afidiíneo faça a oviposição em um hospedeiro, ela deve reconhecê-lo como provável hospedeiro através da avaliação da sua suscetibilidade e qualidade, o que define se esse tem características fisiológicas e nutricionais mínimas para o desenvolvimento das formas jovens do parasitóide. Desta forma, o parasitóide reduz o risco de mortalidade de seus imaturos ao ovipositar em hospedeiros com maior qualidade nutricional. Também, estudos têm demonstrado que $R$. maidis está entre os hospedeiros mais comuns de L. testaceipes (Shufran et al. 2004) e que, em testes com e sem escolha, A. gossypii é o hospedeiro preferido desse parasitóide em relação a Myzus persicae (Sulzer 1776) (Bueno et al. 2003a).

O tempo médio para o desenvolvimento de L. testaceipes em $R$. maidis e A. gossypii foi de 10,2 e 10,1 dias. Carnevale et al. (2003) obtiveram valores inferiores quanto ao

Tabela I. Fecundidade diária $(\overline{\mathrm{X}} \pm \mathrm{EP} *)$ de Lysiphlebus testaceipes nos pulgões Rhopalosiphum maidis e Aphis gossypii. $25 \pm 1^{\circ} \mathrm{C}, \quad \mathrm{UR} 70 \pm 10 \%$ e $12 \mathrm{~h}$ de fotofase.

\begin{tabular}{lccccc}
\hline Hospedeiro & $\begin{array}{c}\text { Idade da fêmea } \\
\text { (dias) }\end{array}$ & $\begin{array}{c}\text { Número de fêmeas } \\
\text { vivas }\end{array}$ & $\begin{array}{c}\text { Pulgões oferecidos/ } \\
\text { fêmea }(\mathrm{n})\end{array}$ & $\begin{array}{c}\text { Total de ovos/ } \\
\text { fêmea }(\overline{\mathrm{X}} \pm \mathrm{EP} *)\end{array}$ & $\begin{array}{c}\text { Pulgões não } \\
\text { parasitados }(\overline{\mathrm{X}} \pm \mathrm{EP} *)\end{array}$ \\
\hline \multirow{4}{*}{ maidis } & 1 & 15 & 300 & $159,6 \pm 8,98$ & $126,0 \pm 8,66$ \\
& 3 & 15 & 250 & $147,8 \pm 8,30$ & $87,1 \pm 10,70$ \\
& 4 & 11 & 200 & $116,6 \pm 10,64$ & $76,2 \pm 10,69$ \\
& 5 & 2 & 150 & $68,2 \pm 16,08$ & $95,8 \pm 10,86$ \\
& 6 & 1 & 100 & $6,0 \pm 2,19$ & $91,0 \pm 1,09$ \\
& 7 & 1 & 50 & $0,00 \pm 0,00$ & $45,0 \pm 0,00$ \\
A. gossypii & 1 & 15 & 50 & $129,00 \pm 0,00$ & $47,0 \pm 0,00$ \\
& 2 & 15 & 250 & $101,1 \pm 16,02$ & $157,2 \pm 13,17$ \\
& 3 & 4 & 200 & $54,9 \pm 13,67$ & $141,9 \pm 15,40$ \\
& 4 & 3 & 150 & $22,2 \pm 13,90$ & $136,1 \pm 7,89$ \\
\end{tabular}

\footnotetext{
*Erro padrão da média
} 
desenvolvimento de L. testaceipes em A. gossypii utilizando algodão como planta hospedeira ( 8,8 dias).

A razão sexual de $L$. testaceipes foi de $0,71 \mathrm{em} R$. maidis e de 0,66 em A.gossypii, sendo esses resultados próximos àqueles obtidos por Rodrigues et al. (2003) em S. graminum $(0,6)$ e por Bueno et al. (2006) em A. gossypii $(0,45)$. Em parasitóides afidiíneos, é comum encontrar um maior número de fêmeas do que de machos na prole, com a razão sexual variando de 0,6 a 0,7 , mas sendo dependente das condições ambientais, do tamanho e da densidade do hospedeiro (Shukla \& Triphathi 1993). Assim, no controle biológico de pulgões, a maior proporção de fêmeas desses parasitóides é extremamente importante, uma vez que elas acasalam uma única vez, enquanto um macho consegue acasalar com várias fêmeas (Hågvar \& Hofsvang 1991). Além disso, segundo Brooijmans \& Van Lenteren (1997), o crescimento populacional de parasitóides é determinado de acordo com o número de fêmeas geradas e o seu período reprodutivo.

Tabela de vida de fertilidade de $L$. testaceipes em $R$. maidis e A. gossypii. As fêmeas do parasitóide L. testaceipes apresentaram comportamento de busca e oviposição assim que foram colocadas em contato com as duas espécies de pulgões hospedeiros testados, sendo que, no primeiro dia de vida da fêmea, foi colocada a maior parte dos ovos nas ninfas hospedeiras de $R$. maidis e A. gossypii (Tab. I). Rodrigues et al. (2003) também observaram maior número de ovos colocados por L. testaceipes no primeiro dia em S. graminum ( 257,8 ovos). Já Steenis (1994) encontrou, para o mesmo parasitóide em $A$. gossypii, cerca de 120 ovos no primeiro dia, valor semelhante ao encontrado neste trabalho.

O parasitóide $L$. testaceipes apresentou uma fecundidade média de 498,2 ovos em $R$. maidis e de 327,8 ovos em $A$. gossypii, e nos três primeiros dias de vida da fêmea, mais de 85 e $87 \%$ dos ovos já haviam sido colocados em $R$. maidis e $A$. gossypii, respectivamente (Fig. 1).

Foi verificada a ocorrência de superparasitismo nas duas espécies de pulgões avaliadas, apesar de a quantidade de ninfas hospedeiras ter sido suficiente para o parasitóide $L$. testaceipes (Tab. II). Observou-se que em cerca de $2 \%$ das ninfas parasitadas de $R$. maidis havia mais do que uma larva por pulgão. Já em A. gossypii o superparasitismo foi de $0,27 \%$. Steenis (1994) obteve $1,34 \%$ de pulgões superparasitados quando L. testaceipes teve A. gossypii como hospedeiro.

A baixa taxa de superparasitismo de $L$. testaceipes nos pulgões avaliados pode estar relacionada à capacidade das fêmeas de afidíneos de discriminar hospedeiros parasitados de não parasitados, o que pode ser feito por meio de marcações químicas no exterior do corpo do hospedeiro na hora da oviposição (Chow \& Mackauer 1986). Substâncias químicas liberadas pela fêmea adulta do parasitóide no momento da oviposição (toque com o ovipositor e colocação do ovo) servem como marcas químicas intraespecíficas, as quais podem ser reconhecidas por contato pelas fêmeas de parasitóides na discriminação dos hospedeiros previamente parasitados. A fêmea é também capaz de discriminar hospedeiros parasitados

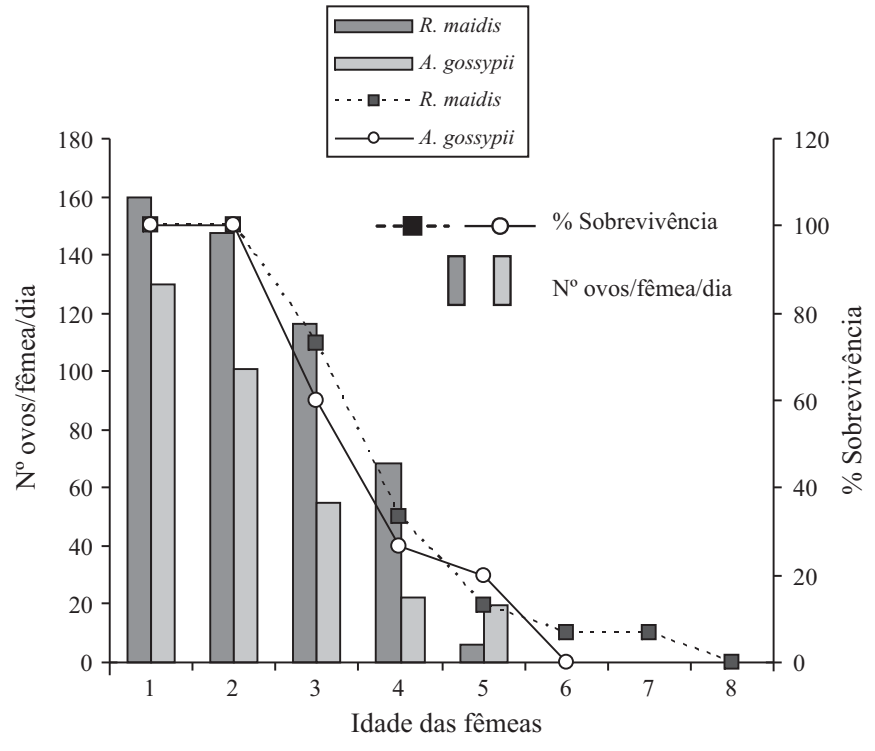

Fig. 1. Produção diária de ovos e porcentagem de sobrevivência da fêmea de Lysiphlebus testaceipes em função da idade em Rhopalosiphum maidis e Aphis gossypii. $25 \pm 1^{\circ} \mathrm{C}$, UR $70 \pm 10 \%$ e $12 \mathrm{~h}$ de fotofase.

a curtas distâncias, por meio das antenas, mesmo 24 horas após o parasitismo, reconhecendo alterações químicas do hospedeiro parasitado causadas pelo desenvolvimento do parasitóide (Medrzycki et al. 2002).

A longevidade média das fêmeas de $L$. testaceipes foi de 3,0 e 2,8 dias quando parasitando $R$. maidis e A. gossypii, respectivamente, e até o terceiro dia, a porcentagem de fêmeas vivas parasitando $R$. maidis foi de $73,6 \%$ e de $60 \%$ em $A$. gossypii (Fig. 1). Rodrigues et al. (2003) observaram valor relativamente maior para a longevidade (4,9 dias) de $L$. testaceipes parasitando $S$. graminum.

Por meio da tabela de vida de fertilidade puderam ser obtidos os parâmetros de crescimento populacional de $L$. testaceipes nos dois pulgões testados, sendo a maior taxa líquida de reprodução $\left(\mathrm{R}_{\mathrm{o}}\right)$ observada em $R$. maidis, quando comparada àquela em A. gossypii. Esses resultados indicam que fêmeas de L. testaceipes têm capacidade de gerar maior número de indivíduos ao longo de sua vida quando são mantidas em $R$. maidis (Tab. III). Esse foi o parâmetro de crescimento populacional que mais se diferenciou entre as espécies de pulgões testadas, tendo sido $25 \%$ maior para $L$. testaceipes mantido em $R$. maidis do que quando o parasitóide foi mantido em A. gossypii.

As taxas intrínsecas de aumento $\left(\mathrm{r}_{\mathrm{m}}\right)$ de L. testaceipes foram de 0,449 quando este foi mantido em $R$. maidis e de 0,431, em A. gossypii (Tab. III). Observa-se que o $\mathrm{r}_{\mathrm{m}}$ de L. testaceipes foi muito próximo para as duas espécies testadas e superiores ao relatado por Steenis (1993) para o parasitóide Aphidius colemani Viereck, $1912\left(\mathrm{r}_{\mathrm{m}}=0,352\right)$ em A. gossypii. Entretanto, Steenis (1994), trabalhando com $L$. testaceipes sobre A. gossypii, obteve $\mathrm{r}_{\mathrm{m}}$ igual a 0,400 , valor semelhante ao do presente trabalho. Já Rodrigues et al. (2003) encontraram, para o mesmo parasitóide, taxa de 0,513 quando em $S$. graminum. 
Tabela II. Número de larvas de Lysiphlebus testaceipes em Rhopalosiphum maidis e Aphis gossypii. $25 \pm 1^{\circ} \mathrm{C}$, UR $70 \pm 10 \%$ e $12 \mathrm{~h}$ de fotofase.

\begin{tabular}{|c|c|c|c|c|c|}
\hline \multirow{2}{*}{ Hospedeiro } & \multirow{2}{*}{$\begin{array}{l}\text { Idade da fêmea } \\
\text { (dias) }\end{array}$} & \multirow{2}{*}{$\begin{array}{l}\text { Número de fêmeas } \\
\text { vivas }(\mathrm{n})\end{array}$} & \multicolumn{3}{|c|}{$\mathrm{N}^{\mathrm{o}}$ de larvas do parasitóide por pulgão $(\overline{\mathrm{X}} \pm \mathrm{EP} *)$} \\
\hline & & & 1 & 2 & 3 \\
\hline \multirow{7}{*}{ R. maidis } & 1 & 15 & $155,9 \pm 8,82$ & $1,9 \pm 0,41$ & $0,00 \pm 0,00$ \\
\hline & 2 & 15 & $140,2 \pm 8,27$ & $3,8 \pm 0,86$ & $0,00 \pm 0,00$ \\
\hline & 3 & 11 & $113,1 \pm 10,44$ & $1,6 \pm 0,42$ & $1,0 \pm 0,08$ \\
\hline & 4 & 5 & $50,6 \pm 14,17$ & $2,0 \pm 1,02$ & $0,00 \pm 0,00$ \\
\hline & 5 & 2 & $6,0 \pm 2,19$ & $0,00 \pm 0,00$ & $0,00 \pm 0,00$ \\
\hline & 6 & 1 & $0,00 \pm 0,00$ & $0,00 \pm 0,00$ & $0,00 \pm 0,00$ \\
\hline & 7 & 1 & $0,00 \pm 0,00$ & $0,00 \pm 0,00$ & $0,00 \pm 0,00$ \\
\hline \multirow{5}{*}{ A. gossypii } & 1 & 15 & $128,4 \pm 12,18$ & $0,7 \pm 0,36$ & $0,00 \pm 0,00$ \\
\hline & 2 & 15 & $100,7 \pm 15,33$ & $0,2 \pm 0,14$ & $0,00 \pm 0,00$ \\
\hline & 3 & 9 & $54,9 \pm 9,35$ & $0,00 \pm 0,00$ & $0,00 \pm 0,00$ \\
\hline & 4 & 4 & $22,2 \pm 2,78$ & $0,00 \pm 0,00$ & $0,00 \pm 0,00$ \\
\hline & 5 & 3 & $19,7 \pm 4,91$ & $0,00 \pm 0,00$ & $0,00 \pm 0,00$ \\
\hline
\end{tabular}

*Erro padrão da média

De acordo com Andrewartha \& Birch (1954), o sucesso de uma espécie em um determinado ambiente é proporcional à sua taxa intrínseca de aumento $\left(\mathrm{r}_{\mathrm{m}}\right)$, ou seja, quanto maior ela for, maior será seu crescimento populacional.

A taxa intrínseca de aumento em parasitóides proporciona tanto informações sobre a taxa de crescimento de sua população como da sua capacidade em reduzir a população da praga, já que, na maioria das vezes, para cada ovo colocado por um parasitóide um hospedeiro é morto (Van Lenteren \& Woets 1988). O conhecimento desses dados também poderá auxiliar no desenvolvimento de métodos de criação massal ou de sistemas de criação aberta para o estabelecimento do parasitóide em casas-de-vegetação (Bennison \& Corless 1993). O pulgão $R$. maidis pode ser, então importante no processo de manutenção de L. testaceipes em sistemas de criação aberta, já que, de acordo com Rabasse \& Steenis (1999), parasitóides afidiíneos, como dos gêneros Aphidius e Lysiphlebus, podem ser mantidos em espécies de pulgões, como $R$. maidis, $R$. padi, $S$. graminum e Sitobion avenae (Fabricius, 1775), os quais se desenvolvem em plantas de trigo, milheto ou cevada, porém, não atacam a cultura de interesse, o que permite a contínua presença dos parasitóides em casa de vegetação.

Em áreas tropicais, parasitóides como L. testaceipes são capazes de colonizar cultivos em casas-de-vegetação e exercer um controle parcial de A. gossypii (Bueno et al. 2003b).

Também se salienta que as características do ciclo de vida de um parasitóide/predador, em comparação com as da praga, são importantes aspectos bioecológicos a serem considerados na avaliação de um inimigo natural potencial para controle biológico; quanto à sua efetividade, o mesmo será considerado efetivo como agente de controle quando apresentar a capacidade de aumentar em número igual ou superior ao da praga (Van Lenterem, 2000). A taxa intrínseca de aumento encontrada por Kuo et al. (2006) para $R$. maidis foi de 0,329 em milho e Soglia et al. (2005) encontraram para A. gossypii 0,310; 0,240 e 0,220, nas cultivares de crisântemo Yellow Snowdon, Dark S. Reagan e White Reagan, respectivamente. Assim, pode-se observar que o parasitóide L. testaceipes apresenta taxa intrínseca de aumento superior as encontradas para as espécies de pulgões hospedeiros, indicando que o parasitóide apresenta potencial para ser utilizado no controle biológico de $R$. maidis e A. gossypii. Isso corrobora estudos realizados por Rodrigues et al. (2005), os quais mostraram, por meio de liberações inoculativas de L. testaceipes para o controle de A. gossypii em duas cultivares de crisântemo (White Reagan e Sunny Reagan) em casa-de-vegetação, que L. testaceipes têm potencial para manter A. gossypii em baixa densidade populacional.

L. testaceipes apresentou uma razão finita de aumento $(\lambda)$ de 1,57 fêmeas/dia em $R$. maidis e de 1,54 fêmeas/dia em $A$. gossypii. O intervalo médio entre gerações (T) foi de 11,86 dias em $R$. maidis e de 11,83 dias em A. gossypii, e o tempo que a população do parasitóide leva para duplicar em número (TD) foi de 10,78 e 11,27 dias em $R$. maidis e A. gossypii, respectivamente (Tab. III). Todos esses parâmetros da tabela de vida de fertilidade de $L$. testaceipes foram muito semelhantes para as duas espécies de pulgões hospedeiros e corroborados com os valores encontrados para o $\mathrm{r}_{\mathrm{m}}$ de L. testaceipes em $R$. maidis e A. gossypii, indicando que o crescimento populacional do parasitóide tende a ser semelhante quando

Tabela III. Parâmetros de crescimento populacional associados a tabela de vida de fertilidade de Lysiphlebus testaceipes em Rhopalosiphum maidis e Aphis gossypii. $25 \pm 1^{\circ} \mathrm{C}$, UR $70 \pm 10 \%$ e fotofase de $12 \mathrm{~h}$.

\begin{tabular}{ccc}
\hline & \multicolumn{2}{c}{ Hospedeiro } \\
\cline { 2 - 3 } Parâmetros de crescimento & R. maidis & A. gossypii \\
\hline $\mathrm{R}_{\mathrm{o}}$ (fêmeas) & 205,38 & 164,08 \\
$\mathrm{r}_{\mathrm{m}}$ (fêmeas/fêmeas/dia) & 0,449 & 0,431 \\
ë(fêmeas/dia & 1,57 & 1,54 \\
$\mathrm{~T}($ dias $)$ & 11,86 & 11,83 \\
$\mathrm{TD}$ (dias) & 10,78 & 11,27 \\
\hline
\end{tabular}

$\mathrm{R}_{\mathrm{o}}=$ taxa líquida de reprodução; $\mathrm{r}_{\mathrm{m}}=$ taxa intrínseca de aumento; $\ddot{\mathrm{e}}=$ razão finita de aumento; $\mathrm{T}=$ tempo médio entre gerações; $\mathrm{TD}=$ tempo de duplicação da população. 
mantido nessas duas espécies de hospedeiros. Esses resultados mostram valores que estão de acordo com aqueles encontrados por Silva et al. (no prelo), os quais reportaram que $L$. testaceipes apresentou maiores taxa de parasitismo e de emergência e maior tamanho quando desenvolvido em $R$. maidis. No entanto, verificaram que o período de desenvolvimento foi menor e a longevidade maior em $A$. gossypii, demonstrando que ambos os hospedeiros apresentam boa qualidade para o parasitóide $L$. testaceipes.

Em função do rápido crescimento das populações dos pulgões, normalmente é essencial introduzir parasitóides no início da sua infestação. Os resultados obtidos nesse estudo evidenciaram que L. testaceipes apresenta capacidade reprodutiva maior do que a dos pulgões hospedeiros estudados, indicando o seu potencial como agente de controle biológico de $R$. maidis e A gossypii. Em função dos dados obtidos, o pulgão $R$. maidis é um hospedeiro indicado para ser utilizado em sistemas de criação aberta para manutenção e conservação de L. testaceipes no interior de casas de vegetação, inclusive para o controle de A. gossypii.

\section{REFERÊNCIAS}

Alves, L. F. A. A.; T. M. V. Prestes; A. Zanini; M. F. Dalmolin \& A. O. Menezes-Jr. 2005. Controle biológico natural de pulgões (Hemiptera: Aphididae) em lavoura de trigo por parasitóides (Hymenoptera, Aphidiinae), no município de Medianeira, PR, Brasil. Semina: Ciências Agrárias 26: 155-160.

Andrewartha, H. G. \& L. C. Birch. 1954. The innate capacity for increase in numbers. In: Andrewartha, H. G. \& L. C. Birch. (Ed.) The distribution and abundance of animals. Chicago: University of Chicago Press, cap. 3, p. 31-54.

Bennison, J. A. \& S. P. Corless. 1993. Biological control of aphids on cucumbers: Further development of open rearing units or "Banker plants" to aid establishment of aphid natural enemies. IOBC/WPRS Bulletin 16: 5-8.

Brooijmans, C. \& J. C. Van Lenteren. 1997. Origins and population dynamics of pest, diseases and weeds. In: J. C. Van Lenteren, (Ed.), Integrated pest management in protected cultivation. Wageningen: Agricultural University Wegeningen, p. 1-16.

Bueno, V. H. P.; A. B. Carnevale \& M. V. Sampaio. 2003a. Host preference of Lysiphebus testaceipes (Cresson) (Hymenoptera: Aphidiidae) for Myzus persicae (Sulzer) and Aphis gossypii Glover (Hemiptera: Aphididae). In: VIII International Symposium on Ecology of Aphidophaga: Biology, Ecology and Behaviour of Aphidophagous Insects, 2003, Ponta Delgada. Proceedings of the 8th International Symposium on Ecology of Aphidophaga. Biology, Ecology, Behaviour of Aphidophagous Insects. Arquipélago: Life and Marine Science, p. 17-20.

Bueno, V. H. P.; J. C. Van Lenteren; L. C. P. Silveira \& S. M. M. Rodrigues. 2003b. An overview of biological control in greenhouse chrysanthemums in Brazil. IOBC/WPRS Bulletin, 26: 1-5.

Bueno, V. H. P. 2005. Controle biológico de pulgões ou afídeos-praga em cultivo protegido. Informe Agropecuário 28: 9-17.

Bueno, V. H. P.; M. V. Sampaio; J. C. Van Lenteren; B. F. Conti; R. J. Silva; S. M. M. Rodrigues \& A. B. Carnevale. 2006. Evaluation of two aphids parasitoids as candidates for biocontrol of aphid pests in protected cultivation in Brazil. IOBC/WPRS Bulletin 29: $175-180$.

Bellows-Junior, T. S.; R. G. Van Driesche \& J. S. Elkinton. 1992. Lifetable construction and analysis in the evaluation of natural enemies Annual Review of Entomology 37: 587-614.

Carnevale, A. B.; V. H. P. Bueno \& M. V. Sampaio. 2003. Parasitismo e Desenvolvimento de Lysiphlebus testaceipes (Cresson) (Hym.: Aphidiidae) em Aphis gossypii Glover e Myzus persicae (Sulser) (Hem.: Aphididae), Neotropical Entomology 32: 293-297.

Carvalho, L. M.; V. H. P. Bueno \& S. M. Mendes. 2006. Ocorrência e flutuação populacional de tripes, pulgões e inimigos naturais em crisântemo de corte em casa de vegetação. Bragantia 65: 139146.

Chow, F. J \& M. Mackauer. 1986. Host discrimination and larval competition in the aphid parasite Ephedrus californicus. Entomologia Experimentalis et Applicata 41: 243-254.

Fonseca, A. R.; I. Cruz; C. F. Carvalho \& B. Souza. 2004. Resistência de genótipos de sorgo ao pulgão Rhopalosiphum maidis (Fitch, 1856) (Hemiptera: Aphididae): III. Efeito no desenvolvimento da planta. Ciência Agrotecnologia 28: 585-592.

Hågvar, E. B. \& T. Hofsvang. 1991. Aphid parasitoids (Hymenoptera, Aphidiidae): biology, host selection and use in biological control. Biocontrol News and Information 12: 13-41.

Kuo, M. H.; M. C. Chiu \& J. J. Perng. 2006. Temperature effects on life history traits of the corn leaf aphid, Rhopalosiphum maidis (Homoptera: Aphididae) on corn in Taiwan. Applied Entomology Zoology 41: 171-177.

Mackauer, M.; J. P. Michaud \& W. Völkl. 1996. Host choice by aphidiid parasitoid (Hymenoptera: Aphidiidae): host recognition, host quality, and host value. Canadian Entomologist 128: 959-980.

Malais, M. H. \& W. J. Ravensberg. 2003. Knowing and recognizing: the biology of glasshouse pests and their natural enemies. Berkel en Rodenrijs, Koppert B.V. 288 p.

Medrzycki, P.; M. Cesari \& S. Maini. 2002. Lysiphlebus testaceipes on Aphis gossypii: studies on remote host discrimination. Bulletin of Insectology 55: 29-33.

Rabasse, J. M. \& M. J. V. Steenis. 1999. Biological control of aphids. In: Albajes, R.; M. A. Gullino; J. C. Van Lenteren \& Y. ELAD. (Ed.) Integrated Pest and Disease Management in Greenhouse Crops, Kluwer Academic Publishers, Dordrecht, The Netherlands, p. $235-243$.

Rochat, J. 1997. Delayed effects in aphid-parasitoid systems: consequences for evaluating biological control species and their use in augmentation strategies. Entomophaga 42: 201-213.

Ramakers, P. M. J. \& M. O’Neill.1999. Cucurbits. In: Albajes, R.; M. A. Gullino; J. C. Van Lenteren \& Y. ELAD. (Ed.) Integrated Pest and Disease Management in Greenhouse Crops, Kluwer Academic Publishers, Dordrecht, The Netherlands, p. 435-453.

Ramakers, P. M. J. \& R. H. M. Maaswinkel. 2002. Pest occurrence and control in organic year-round production of chrysanthemums. IOBC/WPRS Bulletin 25: 221-224.

Rodrigues, S. M. M.; V. H. P. Bueno \& J. S. S. Bueno Filho. 2001. Desenvolvimento e avaliação do sistema de criação aberta no controle de Aphis gossypii Glover (Hem.: Aphididae) por Lysiphlebus testaceipes (Cresson, 1880) (Hym.: Aphidiidae) em casa-devegetação. Neotropical Entomology 30: 433-436.

Rodrigues, S. M. M. \& V. H. P. Bueno. 2001. Parasitism rate of Lysiphlebus testaceipes (Cresson) (Hym.: Aphidiidae) on Schizaphis graminum (Rond.) and Aphis gossypii Glover (Hem.: Aphididae). Neotropical Entomology 30: 625-629.

Rodrigues, S. M. M.; V. H. P. Bueno \& M. V. Sampaio. 2005. Efeito da liberação inoculativa sazonal de Lysiphlebus testaceipes (Hym., Braconidae, Aphidiinae) na população de Aphis gossypii Glover (Hemiptera, Aphididae) em cultivo de crisântemo em casa de vegetação comercial. Boletin de Sanidad Vegetal-Plagas 31: 367-374.

Rodrigues, S. M. M.; V. H. P. Bueno; M. V. Sampaio \& M. C. M. Soglia. 2004. Influência da temperatura no desenvolvimento e parasitismo de Lysiphlebus testaceipes (Cresson) (Hymenoptera: Braconidae, Aphidiinae) em Aphis gossypii Glover (Hemiptera: Aphididae). Neotropical Entomology 33: 341-346.

Rodrigues, S. M. M.; V. H. P. Bueno \& M. V. Sampaio. 2003. Tabela de vida de fertilidade de Lysiphlebus testaceipes (Cresson, 1880) (Hymenoptera, Aphidiidae) em Schizaphis graminum (Rondadni, 1852) (Hemiptera, Aphididae). Revista Brasileira de Entomologia 47: 637-642. 
Sequeira, R. \& M. Mackauer. 1993. The nutricional ecology of a parasitoid wasp, Ephedrus californicus Baker (Hymenoptera: Aphidiidae). Canadian Entomologist 125: 423-430.

Shufran, K. A.; A. A. Weathersbee; D. B. Jones \& N. C. Elliott. 2004. Genetic similarities among geographic isolates of Lysiphlebus testaceipes (Hymenoptera: Aphidiidae) differing in cold temperature tolerances. Environmental Entomology 33: 776-778.

Shukla, A. N. \& C. P. M. Triphathi. 1993. Effect of food plants on the offspring sex ratio of Diaeretiella rapae (Hymenoptera: Aphidiidae), a parasitoid of Lipaphis erysimi Kalt. (Hemiptera: Aphididae). Biology, Agriculture and Horticulture 9: 137146.

Silva, R. J; V. H. P. Bueno \& M. V. Sampaio. Qualidade de diferentes espécies de pulgões como hospedeiros ao Parasitóide Lysiphlebus testaceipes (Cresson) (Hymenoptera, Braconidae, Aphidiinae), Neotropical Entomology (no prelo).

Soglia, M. C. M.; V. H. P. Bueno \& M. V. Sampaio. 2005. Fertility life table of Aphis gossypii on three commercial chrysanthemum cultivars. IOBC/WPRS Bulletin 28: 241-244.

Soglia, M. C. M.; V. H. P. Bueno; M. V. Sampaio; S. M. M. Rodrigues \& C. A. S. Ledo. 2006. Desenvolvimento e parasitismo de Lysiphlebus testaceipes (Cresson) e Aphidius colemani Viereck (Hymenoptera:
Braconidae) em Aphis gossypii Glover (Hemiptera: Aphididae) em duas cultivares de crisântemo. Neotropical Entomology 35: 364 370 .

Starý, P.; M. V. Sampaio \& V. H. P. Bueno. 2007. Aphid parasitoids (Hymenoptera, Braconidae, Aphidiinae) and their associations related to biological control in Brazil. Revista Brasileira de Entomologia 51: 107-118.

Steenis, M. J. V. 1993. Intrinsic rate of increase of Aphidius colemani Viereck (Hym.; Braconidae), a parasitoid of Aphis gossypii Glover (Hom., Aphididae) at different temperatures. Journal of Applied Entomology 116: 192-198.

Steenis, M. J. V. 1994. Intrinsic rate of increase of Lysiphlebus testaceipes (Cresson) (Hym.; Braconidae), a parasitoid of Aphis gossypii Glover (Hom., Aphididae) at different temperatures. Journal of Applied Entomology 118: 399-406.

Van Lenteren, J. C. 2000. Critérios de seleção para avaliação de inimigos naturais em controle biológico. In: Bueno, V. H. P. (ed.). Controle Biológico de Pragas: produção massal e controle de qualidade. Lavras, (Ed.) UFLA, p. 1-19.

Van Lenteren, J. C. \& J. Woets. 1988. Biological and integrated pest control in greenhouses. Annual Review of Entomology 33: 239269.

Recebido em 03/05/2007; aceito em 14/01/2008 